\title{
Examination of Hydrate Formation Methods: Trying to Create Representative Samples
}

\author{
Timothy J. Kneafsey \\ Emily V.L. Rees \\ Seiji Nakagawa \\ Tae-Hyuk Kwon \\ Lawrence Berkeley National Laboratory
}

June 1, 2010

This work was supported by the U.S. Department of Energy and LBNL under Contract No. DE-AC02-05CH11231. 


\section{Examination of Hydrate Formation Methods: Trying to Create Representative Samples}

Timothy J. Kneafsey, Emily V.L. Rees, Seiji Nakagawa, and Tae-Hyuk Kwon

Lawrence Berkeley National Laboratory

\section{Introduction}

Forming representative gas hydrate-bearing laboratory samples is important so that the properties of these materials may be measured, while controlling the composition and other variables. Natural samples are rare, and have often experienced pressure and temperature changes that may affect the property to be measured [Waite et al., 2008].

Forming methane hydrate samples in the laboratory has been done a number of ways, each having advantages and disadvantages. The ice-to-hydrate method [Stern et al., 1996], contacts melting ice with methane at the appropriate pressure to form hydrate. The hydrate can then be crushed and mixed with mineral grains under controlled conditions, and then compacted to create laboratory samples of methane hydrate in a mineral medium. The hydrate in these samples will be part of the load-bearing frame of the medium. In the excess gas method [Handa and Stupin, 1992], water is distributed throughout a mineral medium (e.g. packed moist sand, drained sand, moistened silica gel, other porous media) and the mixture is brought to hydrate-stable conditions (chilled and pressurized with gas), allowing hydrate to form. This method typically produces graincementing hydrate from pendular water in sand [Waite et al., 2004]. In the dissolved gas method [Tohidi et al., 2002], water with sufficient dissolved guest molecules is brought to hydrate-stable conditions where hydrate forms. In the laboratory, this is can be done by pre-dissolving the gas of interest in water and then introducing it to the sample under the appropriate conditions. With this method, it is easier to form hydrate from more soluble gases such as carbon dioxide. It is thought that this method more closely simulates the way most natural gas hydrate has formed. Laboratory implementation, however, is difficult, and sample formation is prohibitively time consuming [Minagawa et al., 2005; Spangenberg and Kulenkampff, 2005]. In another version of this technique, a specified quantity of gas is placed in a sample, then the sample is flooded with water and cooled [Priest et al., 2009].

We have performed a number of tests in which hydrate was formed and the uniformity of the hydrate formation was examined. These tests have primarily used a variety of modifications of the excess gas method to make the hydrate, although we have also used a version of the excess water technique. Early on, we found difficulties in creating uniform samples with a particular sand/ initial water saturation combination (F-110 Sand, $\sim 35 \%$ initial water saturation). In many of our tests we selected this combination intentionally to determine whether we could use a method to make the samples uniform.

The following methods were examined:

-Excess gas

-Freeze/thaw/form

-Freeze/pressurize/thaw 
-Excess gas followed by water saturation

-Excess water

- Sand and kaolinite

-Use of a nucleation enhancer (SnoMax)

-Use of salt in the water

Below, each method, the underlying hypothesis, and our results are briefly presented, followed by a brief conclusion. Many of the hypotheses investigated are not our own, but were presented to us. Much of the data presented is from x-ray CT scanning our samples. The x-ray CT scanner provides a three-dimensional density map of our samples. From this map and the physics that is occurring in our samples, we are able to gain an understanding of the spatial nature of the processes that occur, and attribute them to the locations where they occur.

\section{Methods}

\section{Partially Saturated Porous Media-Moist Sand (Excess Gas Method)}

\section{Description}

In this method, water-wetting sand is uniformly moistened and then packed into a vessel or sleeve. The void space is evacuated, and the hydrate-forming gas is introduced. Hydrate is formed either by pressurizing the system well into the stability zone and then cooling, or by pressurizing the already chilled sample. In either case, heat released from hydrate formation must be absorbed by the system. In our tests this will result in a temperature increase up to the hydrate stability point at the imposed pressure, and heat transfer through the rubber sleeve or vessel wall to the temperature controlled fluid.

\section{Hypothesis}

Hydrate formation will be relatively uniform because the water is evenly distributed in the sample. Some increased hydrate saturations will occur at the outer radii due to better heat transfer there. The hydrate formed will cement the mineral grains together as observed by Waite et al., (2004)

\section{Results}

This technique has been used in both natural porous media and homogeneous and heterogeneous laboratory sand packs. Hydrate has been formed both by cooling pressurized systems, and pressurizing cooled systems. The duration of the incubation time, in which methane dissolves into the water and hydrate nucleation occurs, is unpredictable. In many cases, it has been sufficiently long such that the system sits isothermally within the hydrate stability zone for some time (tens of hours) prior to hydrate forming. This method produced both well-distributed hydrate and very heterogeneous hydrate. A few examples are presented in Figure 1. 


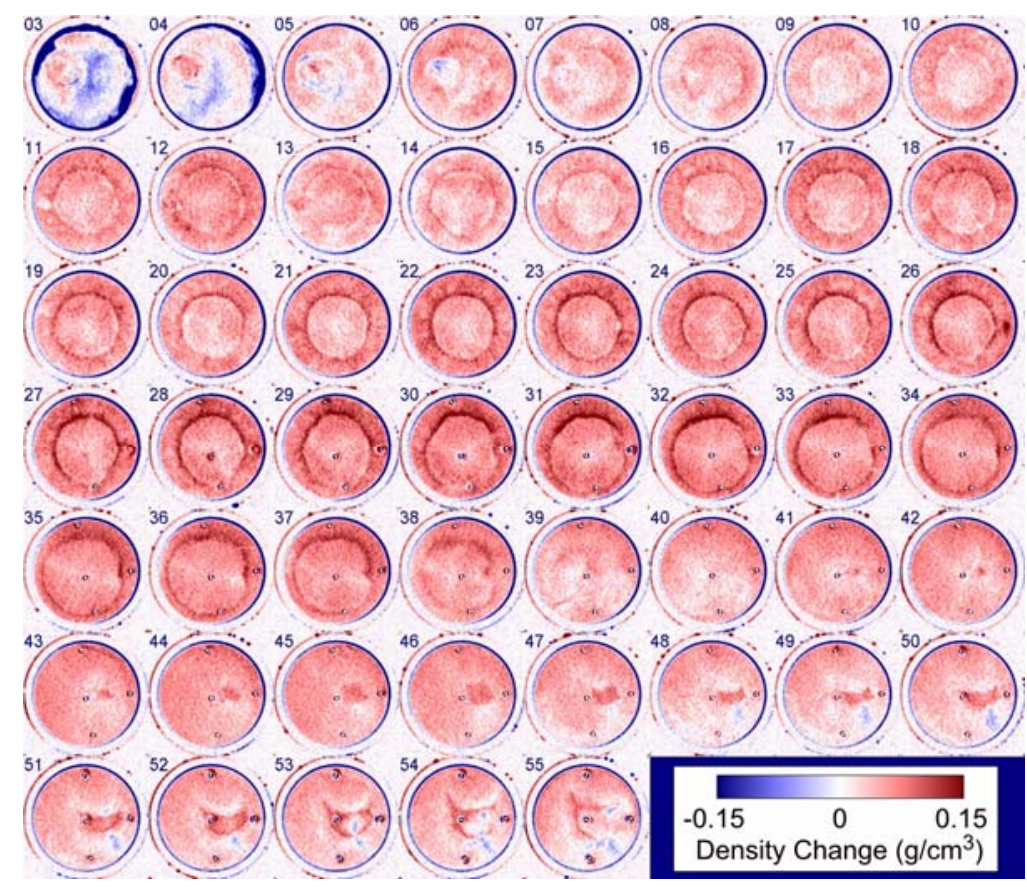

a.

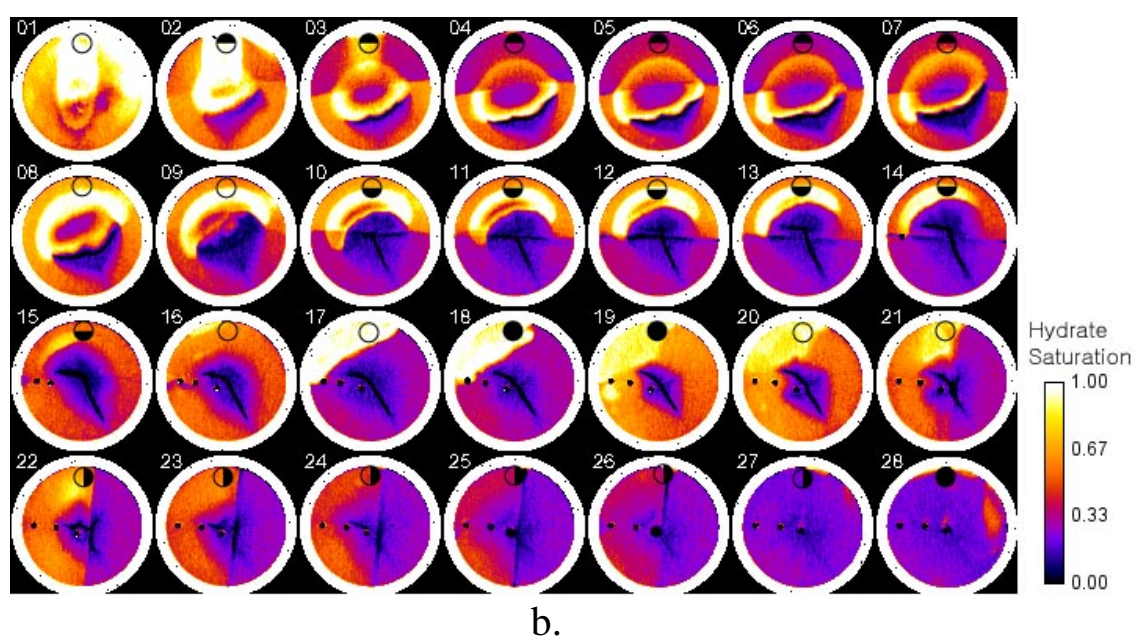



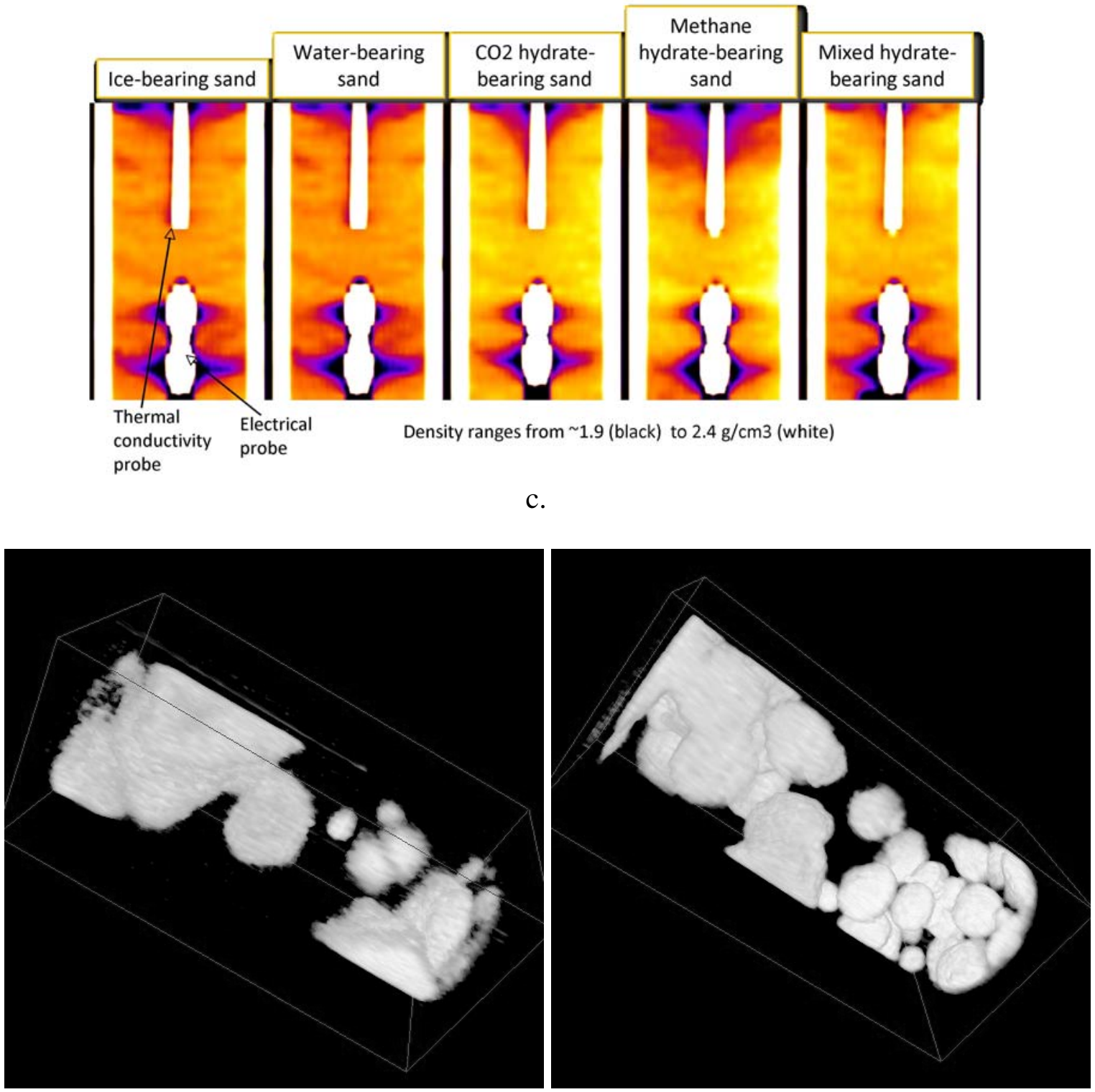

d.

Figure 1. X-ray CT scan data showing a. Density change in a sample upon hydrate formation showing increased density changes (red) from hydrate formation at high porosity locations [Kneafsey et al., 2007], b. Heterogeneous hydrate saturation in a heterogeneously packed sample [Seol and Kneafsey, 2009] (Note: heterogeneity in hydrate saturation is affected by the initial sample heterogeneity, but not dominated by it), and c. Three hydrate formation experiments in a packed sand sample. Density ranges from $\sim 1.9$ (black) to $2.4 \mathrm{~g} / \mathrm{cm}^{3}$ (white), d. Laboratory-formed hydrate locations (white) in a natural core; left - hydrate by cooling, right - hydrate by pressurization.

\section{Conclusions}

Hydrate begins to form in specific locations. These locations are governed by the conditions at those locations at the time hydrate starts to from, and the stochastic nature of nucleation. Hydrate present in porous media alters the capillary strength of the 
medium, drawing water towards itself [Gupta et al., 2006; Kneafsey et al., 2007]. The hydrate continues to form outward from the starting locations, continuing the process. The result is hydrate that may be quite nonuniform.

\section{Partially Saturated Porous Media-Moist Sand and Moist Sand with Silt - Freeze/Thaw/Form Hydrate}

\section{Description}

This method is similar to the excess gas method described above except that the sample is frozen and thawed prior to bringing the sample into the hydrate stability zone.

\section{Hypothesis}

Freezing and thawing water provides nucleation "seeds" abundantly throughout the sample. The prevalence of these seeds will cause hydrate to start forming in very many locations (everywhere) at once.

\section{Results}

A number of tests were performed using this technique. Two different sands (US Silica F-110 silica sand, and "K-sand - an angular silica sand having slightly larger grains than the F-110) and a sand-silt mixture (90\% F-110 and 10\% US Silica Sil-Co-Sil 45) were used.

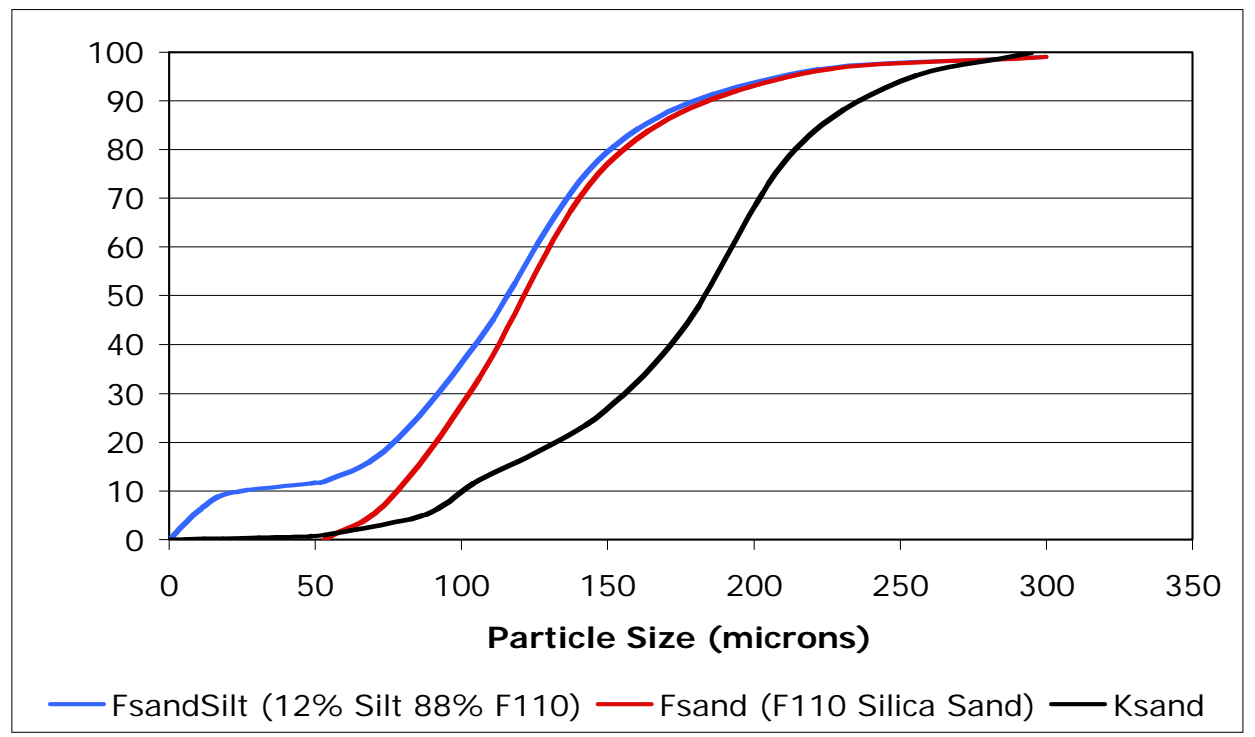

a. 


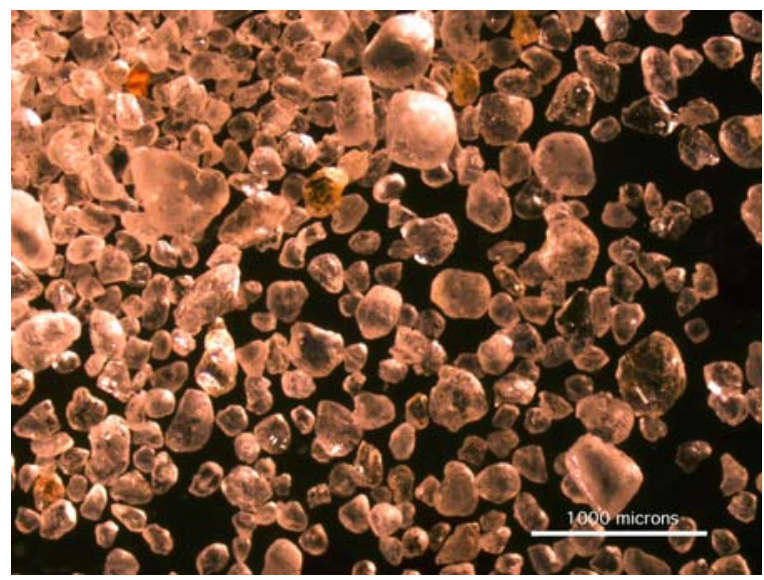

b.

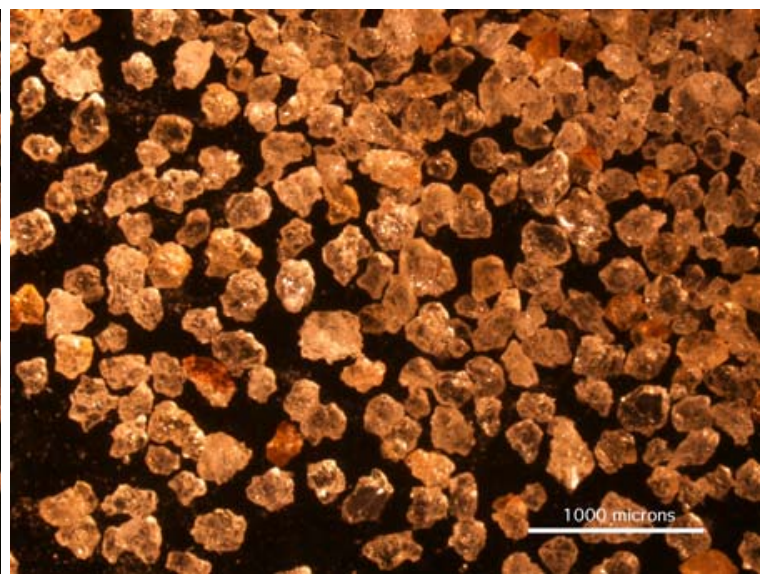

C.

Figure 2. a. Sieve analysis of media used, b. photomicrograph of F-110 sand, c. photomicrograph of K-sand.

In the tests, $\mathrm{x}$-ray CT scans of the initial moist sample, frozen sample, thawed sample, and hydrate-bearing sample were obtained. Figure 3 shows averages of the CT scans obtained for each sample. For each porous medium, twelve averaged images are shown. In each horizontal row, there are four conditions: sand/water/nitrogen (S/W/N), sand/ice/nitrogen (S/I/N), sand/water/nitrogen (S/W/N), and sand/hydrate/methane $(\mathrm{S} / \mathrm{H} / \mathrm{M})$. The data shown are density values, however the scales were selected to examine the sample structure. In each sample, higher density from better packing and higher water saturation is brighter than lower saturation. Additionally, each sample is shown with the density calibration scaled to that particular test. Because of this, the lower moisture content samples appear as bright as the higher moisture content samples, despite the higher moisture content samples having a higher density.

Figure 3a shows results for the F-110 sand. For the low initial water saturation case ( $20 \%$ ), the initial water saturation was slightly greater in the sample center indicating slightly better packing there. Freezing the sample tended to draw the moisture to the outer radii of the sample. This is because freezing would occur from the outside towards the inside, and ice filling the pore space at the outer radii would increase the capillary suction there drawing water towards the outer radii. Thawing the sample resulted in a fairly uniform water saturation. Hydrate formation resulted in a concentration of hydrate in the sample center. For the medium initial water saturation ( 35), the initial moisture distribution was fairly constant. Freezing had the same effect as it did for the lower initial water saturation case and concentrated the ice towards the outer radii. Thawing redistributed the water evenly, but hydrate formation resulted in a very heterogeneous hydrate distribution. In the highest initial water saturation case ( 50\%), with the exception of a spot in the center, the same trends were observed as in the low initial water saturation case.

For the Ksand (Figure 3b) the trends are similar, with a few differences. Freezing the lowest initial water saturation case resulted in little change. This is probably due to the lower packing density, and the roughness of the grains increasing their ability to hold water. Hydrate formation resulted in higher hydrate saturation in the sample center. For 
the higher initial water saturation cases, the effect of freezing was greater with water moving outward, and hydrate formation was preferential in the sample center resulting in high saturation there.

For the F-110/silt mixture, the results were similar in nature to the Ksand results with freezing causing an increase in saturation at the outer radii of the sample, and hydrate formation occurring throughout the sample, but preferentially along the center axis.

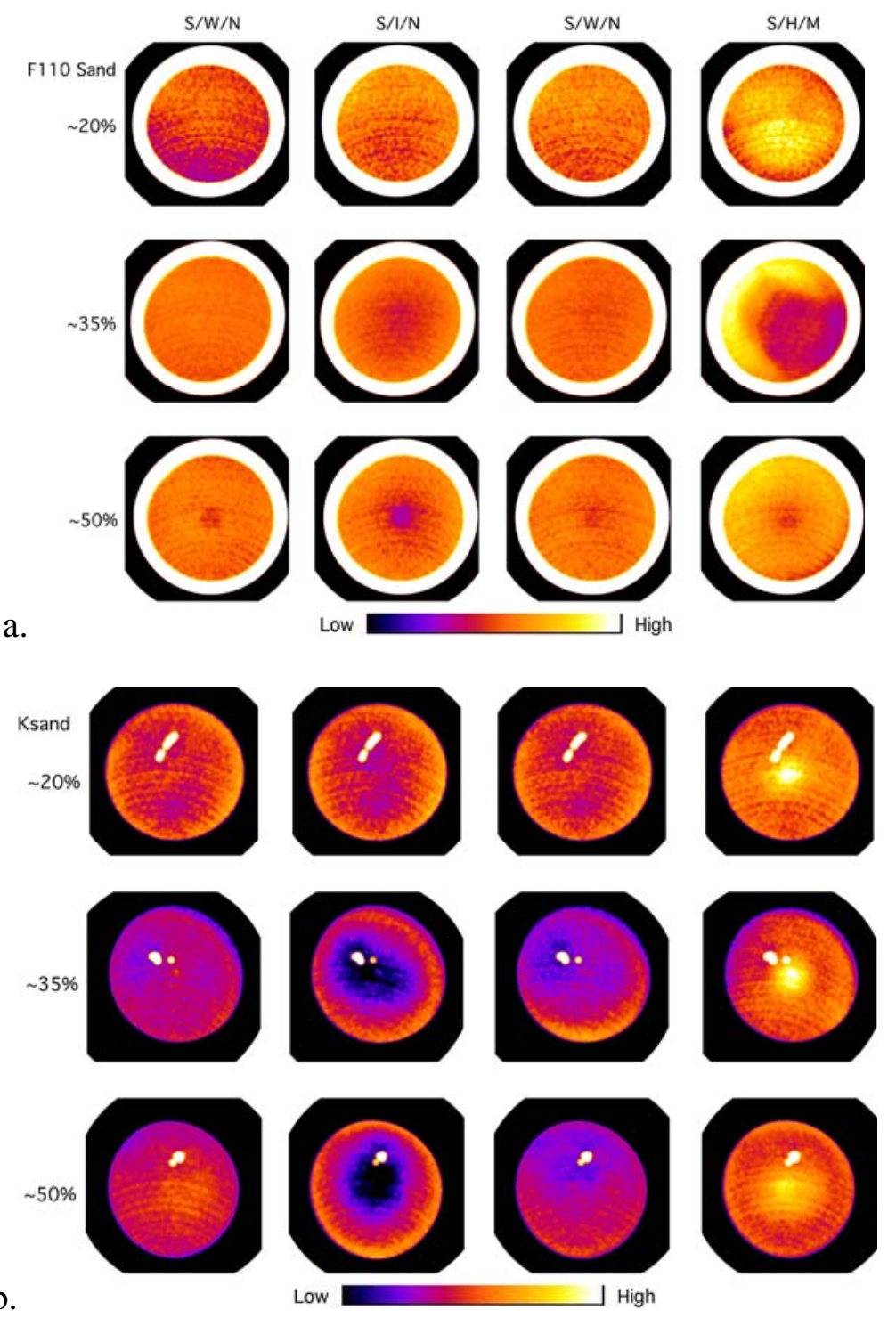



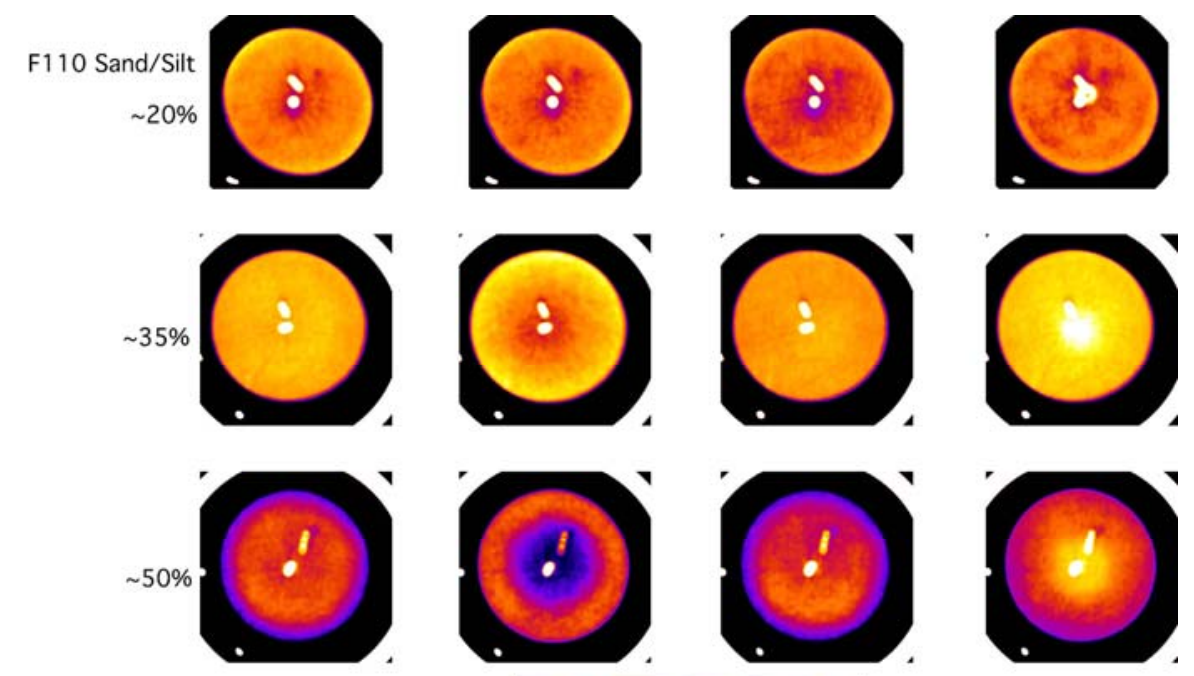

c.

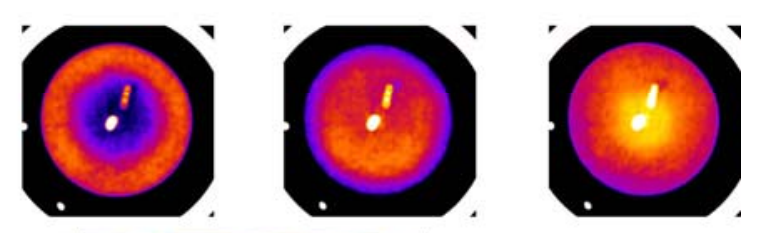

Low

$\checkmark$ High

Figure 3. Average sample densities for three porous media (a. F-110 Sand, b. Ksand, and c. F110/Silt mixture) at three initial water saturations (rows in each subfigure) and under three conditions (moist [SWN], frozen [SIN], thawed [SWN], and hydrate bearing [SHM]). Each image is the average of the cross sections (up to 54) for the particular test.

Density profiles through each of the plots of frozen and hydrate-bearing samples shown in Figure 3 are presented in Figure 4. In each of these plots, the sample density for the frozen samples is presented in black, with the thickness of the line being in proportion to the initial water saturations (thicker lines for higher initial water saturations), and the hydrate bearing samples are plotted in red also with the thickness of the line being in proportion to the initial water saturations. In all cases, the average density of the sample increases upon hydrate formation from the incorporation of methane into the hydrate. For the F-110 sand (Figure 4b) at the lowest initial water saturation, the change from frozen to hydrate bearing shows a dramatic increase in the center. For the very heterogeneous moderate initial water saturation case, there is relatively more hydrate forming at the outer radii (for this profile location), and for the highest initial water saturation case, the density in the center is low for both ice and hydrate-bearing samples, however there is relatively more hydrate in the center than ice. For both the Ksand and F-110 sand/silt media, the density trends of the ice-bearing samples and hydrate-bearing samples are opposite. The density of the ice-bearing samples is higher at the outer radii, whereas the density of the hydrate-bearing samples is higher in the center. 


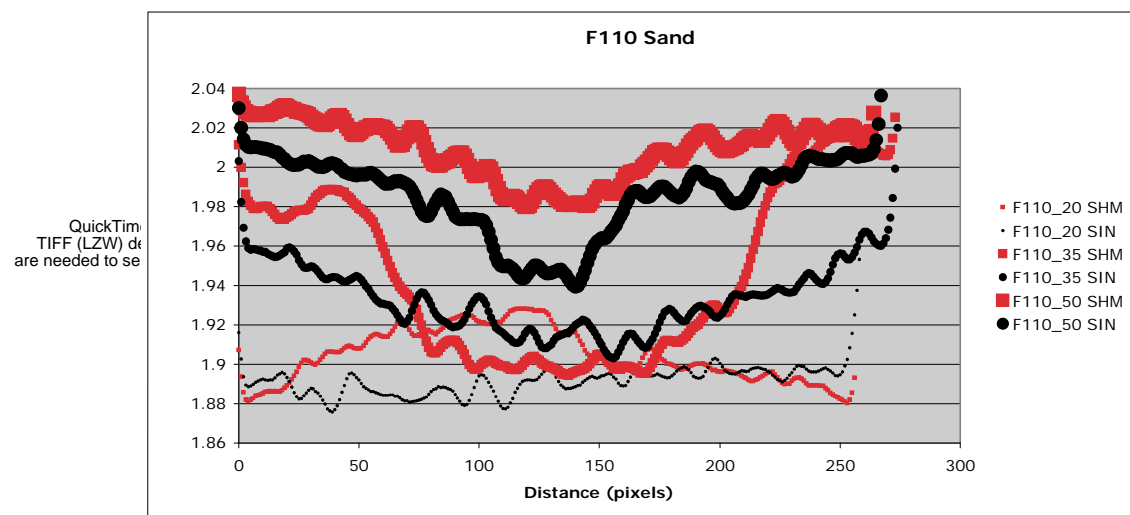

a.

b.

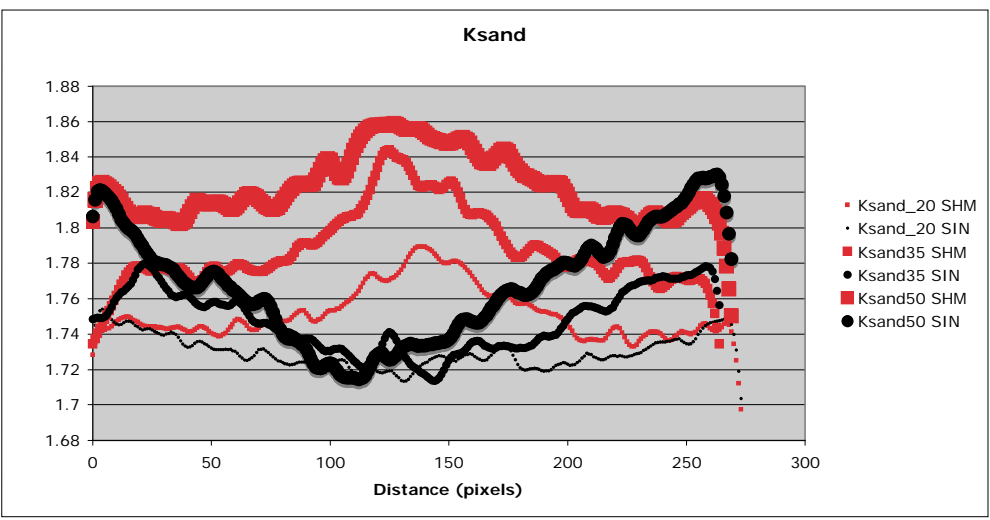

C.

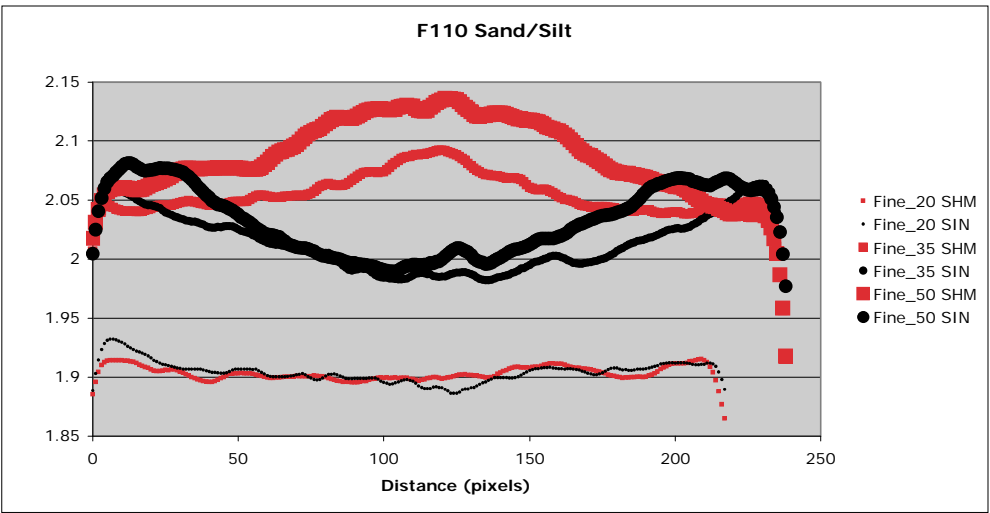

d.

Figure 4. a. Profile location, and sample density across the profile for b. F-110 Sand, c. Ksand, and d. F-110 Sand/Silt.

\section{Conclusions}

The "typical" behavior of the systems investigated was that freezing attracted water towards the freezing front, which in these samples was at the outer radius. This can be explained by the combination of two phenomena. The first of these is that as ice forms, it makes the pores smaller inducing flow by capillarity. The second is that where the water freezes, there is a lower energy, causing an energy gradient driving water to that location. Water behavior in hydrate-bearing systems should be governed by the same principles, 
yet hydrate formation was typically concentrated in the sample center, where heat transfer is the poorest. With the exception of the F-110 sand at 35\% initial water saturation, gradients in hydrate saturation are not severe, however the resulting saturations cannot be considered uniform.

\section{Unsaturated - Freeze/Pressurize/Thaw}

\section{Description}

In this test, moist F-110 sand was packed into a sleeve contained in a pressure vessel. A confining pressure was applied to the sample, and the sample was chilled until it froze. At that point, the sample was evacuated, and pressurized with methane. The temperature was allowed to warm through the melting point to allow melting and ultimately reached $3^{\circ} \mathrm{C}$.

\section{Hypothesis}

Melting ice will provide hydrate "seeds" for nucleation. Hydrate will form in the same location as the melting ice when the ice is melted in the presence of methane under hydrate-stable conditions. Freezing water in a porous medium will result in changes in the sample density from changes in capillarity. These changes can be moderate, compared to some observed for hydrate formation.

\section{Results}

Hydrate formation did not occur immediately upon thawing, thus water had the ability to move upon hydrate formation. This resulted in a heterogeneous hydrate saturation distribution. Figure 5 shows $3 \mathrm{~mm}$ thick CT cross-sections from every $12 \mathrm{~mm}$ over the sample showing hydrate distribution pattern. The red color indicates increases in sample density resulting from hydrate formation. The blue color indicates a decrease in density from water migration away from those locations.

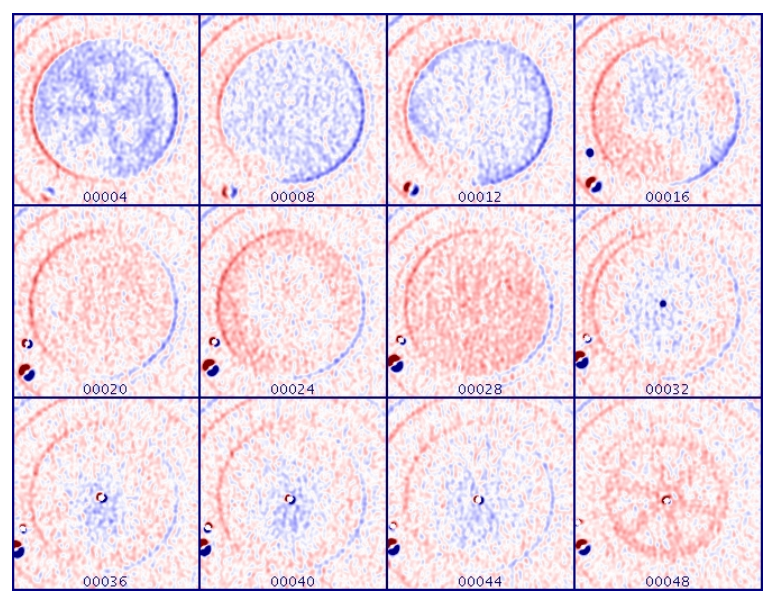

a.

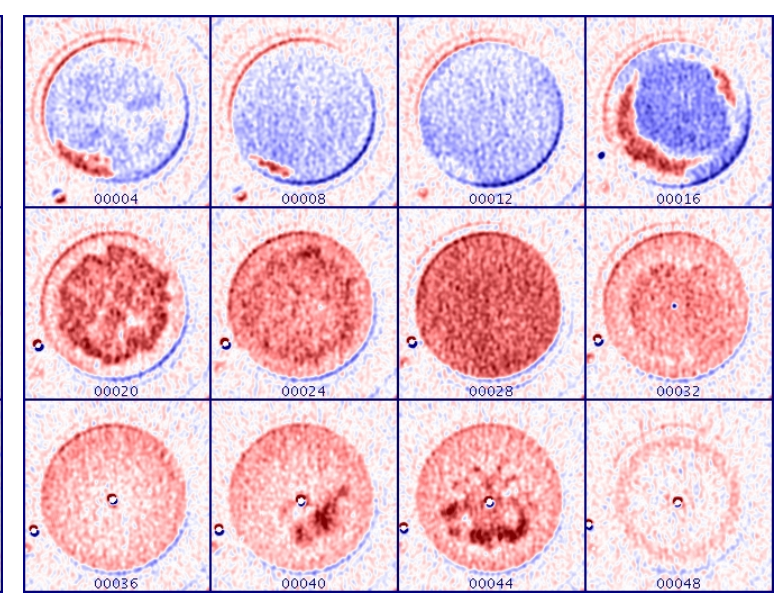

b.

Figure 5. Density changes from moist sand upon a. freezing, and b. hydrate formation. Red shows increase in density, blue shows decrease. The maximum increase and decrease for freezing is about $0.07 \mathrm{~g} / \mathrm{cm}^{3}$, and for hydrate formation the maximum is approximately $0.2 \mathrm{~g} / \mathrm{cm}^{3}$, whereas the maximum decrease is about $0.11 \mathrm{~g} / \mathrm{cm}^{3}$.

\section{Conclusions}


As observed in other tests, freezing alters the moisture distribution. This will be time dependent as moisture migration and heat transfer are both depend on time. More rapid freezing could result in more uniform ice distribution. If hydrate does not form immediately upon thawing and the temperature is allowed to rise, water will be able to move. Very slow melting could be the solution to this problem. Combining these two solutions may result in uniformly distributed hydrate.

\section{Unsaturated - Form Hydrate Using Excess Gas Method Followed by Water Saturation}

\section{Description}

In this method, moist sand is packed into a sleeve or pressure vessel, briefly evacuated, and then brought into the hydrate stability zone by a combination of pressurization and cooling (excess gas method). Following the hydrate formation, water is injected into the sample and the remaining gas is bled out simultaneously. Some trapped gas remains, and the system pressure is maintained with water until water is no longer taken into the sample.

\section{Hypothesis}

Even for a dilute solution, ions will be concentrated near the water-mineral interface as described by the diffuse double layer theory. Hydrate formation excludes ions, thus as hydrate forms, the concentration of ions in the remaining water increases. In addition, ions hinder hydrate formation and the hydrate stability zone is shifted by the salt concentration. Water held in an unsaturated water-wetting porous medium will be in films and pendular structures at grain contacts. At the pore scale using the excess gas method, hydrate should form at those locations as well, however formation immediately adjacent to the mineral surface is unlikely because of the concentration of ions there. In this method, the hydrate forms with a large surface area near a "concentrated" ionic solution. Replacing the excess gas with water after hydrate formation dilutes the ionic concentration, and allows the hydrate to reduce its surface area thus minimizing free energy. This minimization and the tendency for hydrate to move away from the diffuse double layer will result in hydrate crystals in the pore bodies.

\section{Results}

Using a Split Hopkinson Resonant Bar (SHRB) method, seismic resonance frequency and attenuation of our sample was measured (1) prior to hydrate formation (moist sand), (2) during hydrate formation, (3) during the water flood immediately following the hydrate formation, and, finally, (4) during hydrate dissociation. Through numerical inversions, the measured data were used to determine the compression (P-) and shear (S-) wave velocities.

Figure 6 shows the measured compression and torsion mode resonance frequencies over the duration of the test, Note that these are for the fundamental mode resonances of the SHRB test assembly, which are sensitive to the changes in the sample's Young's modulus (E) and shear modulus (G), respectively. Also note that these modes are not sensitive to small changes in the density of the sample. The sample becomes much stiffer 
upon hydrate formation (cementing). The stiffness increases somewhat over the next 100 hours or so, and the resulting P and S-wave velocities increase as well. Water injection causes the resonant frequency to decline significantly, yet the resulting frequenciesparticularly the torsion mode frequencies-are still above the starting (moist sand) frequencies. These changes indicate that the hydrate within the sand pack changes its habit during water flooding: the initial, grain-cementing hydrate becomes pore-filling hydrate, resulting in reduced frame-stiffening effect. Thermal dissociation causes the sample to lose stiffness further, close to the initial (moist sand) condition at the end of the experiment.

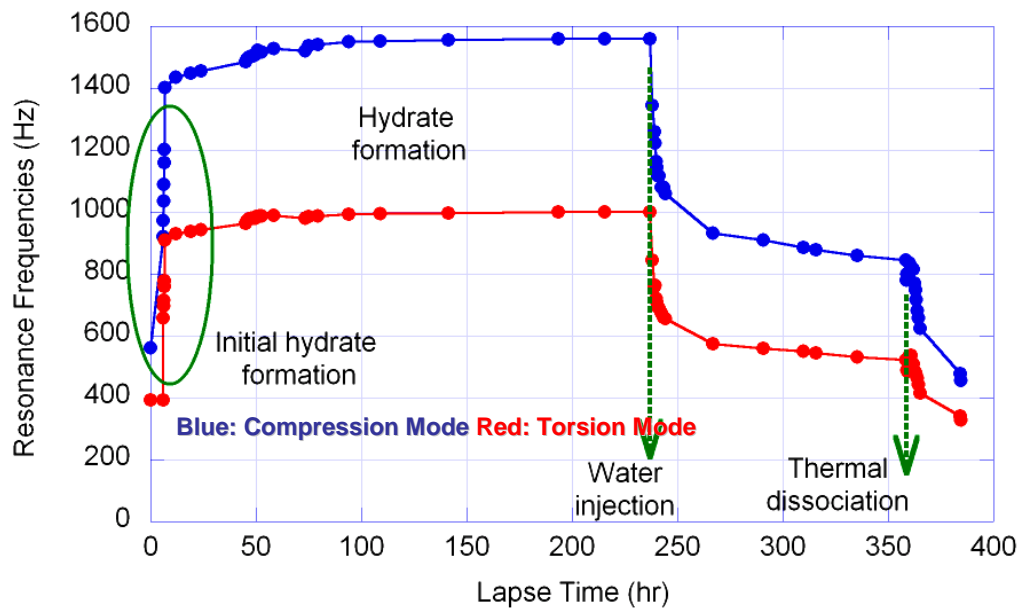

Figure 6. Evolution of measured resonance frequencies during the experiment (Test\#1).

Figure 7 presents the seismic velocities and attenuation for two water injection experiments. In Test 1, water was easily injected into the sample, whereas in Test 2, water was only slowly injected because of hydrate formation and pore space and line clogging. In both tests however, the seismic velocities decreased significantly (up to $\sim 50 \%$ of the initial velocities) in spite of the probable formation of additional hydrate in the samples from the addition of water. The attenuation rapidly increased upon water injection. These increases are probably caused by the formation of new hydrate crystals within the sample. The seemingly contradicting results-formation of additional hydrate and decreasing seismic velocities (i.e., cementation between sediment grains) — are attributed to the migration of hydrate crystals from intergranular contacts to the pore fluid. This explanation is supported by the observation by Katsuki et al (2006, 2007). Using glass micromodels, they showed that when water-saturated, gas hydrates (methane hydrate and $\mathrm{CO}_{2}$ hydrate) change their habit within the pore space over time. 

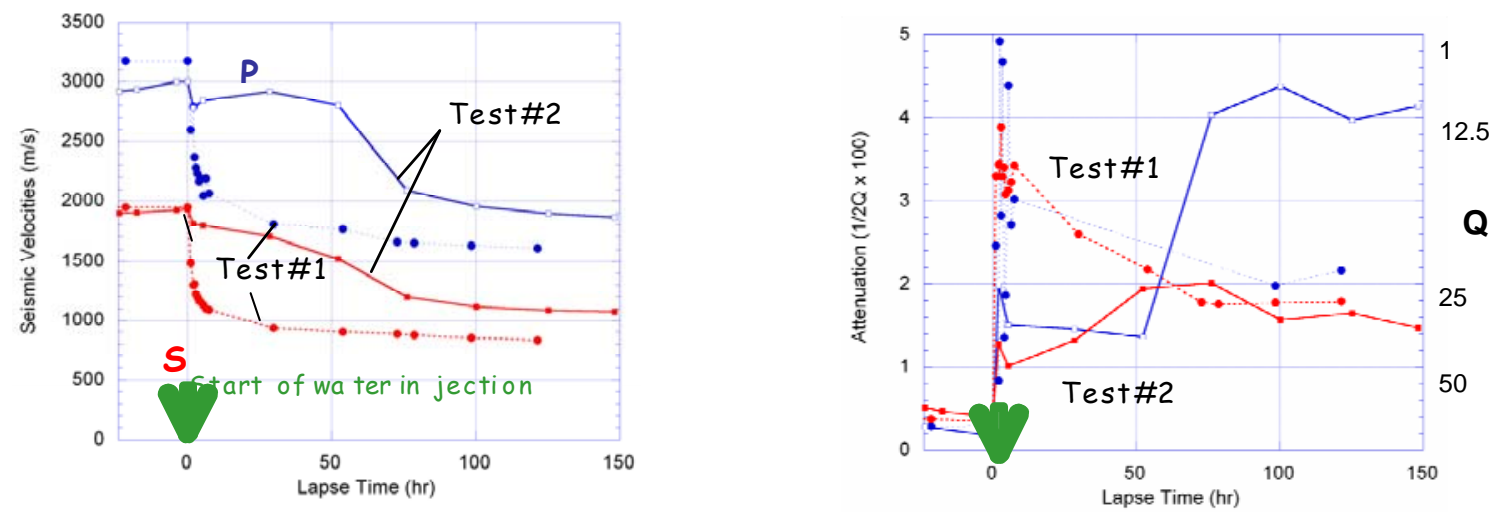

Figure 7. Seismic velocities and attenuation over the water injection period. Hydrate clogging in Test 2 resulted in slow uptake of water.

\section{Conclusions}

The introduction of water into a sand pack containing grain-cementing hydrate resulted in a significant reduction of the sample stiffness (both Young's and shear moduli and P and $S$ velocities). This indicates debonding of hydrate from the mineral grains. The resulting stiffness of the sample, however, was still higher than the moist sand sample it was formed from, which is also consistent with the behavior of a sample containing hydrate occupying the pore bodies [Yun et al., 2007].

\section{Excess Water Method (Southampton Method)}

\section{Description}

A predetermined quantity of methane is added to dry sand sample in a sleeve or pressure vessel. Water is then added at the hydrate formation pressure and the sample is cooled. Hydrate formation consumes methane, and water is continuously added at the formation pressure.

\section{Hypothesis}

Water imbibition occurs uniformly throughout the sample. Gas is trapped in the large pore bodies within the sand as the water is imbibed. Hydrate formation occurs in these pore bodies resulting in pore body-filling hydrate.

\section{Results}

During sample preparation, a high porosity zone was created near the thermocouple. Initially upon waterflooding, the sample did not imbibe water uniformly particularly in this region, and a gas-rich zone was formed there (See Figure 8). Upon hydrate formation, the density increased significantly in the gas-rich zone. This is probably a result of two processes; hydrate formation, and water imbibition. In the sample region that was initially well saturated (indicated by an oval in Figure 8), very little additional change occurred upon hydrate formation. Larger increases in density from the same two processes can be seen in the upper left quadrant, which was partially water saturated during the water flood. 

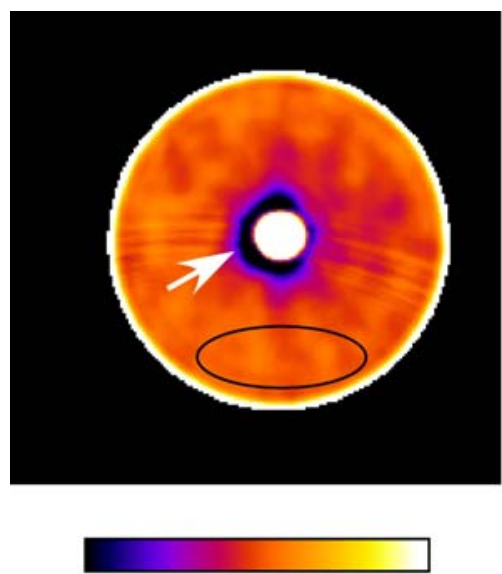

1.851 .952 .052 .152 .25

Density $\left(\mathrm{g} / \mathrm{cm}^{3}\right)$
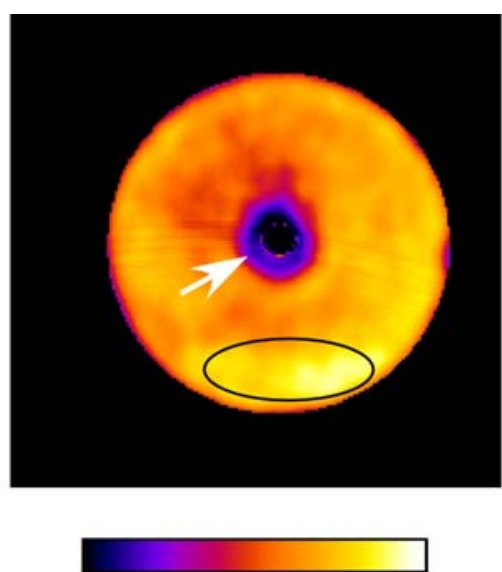

0.000 .100 .200 .300 .40

Density Change $\left(\mathrm{g} / \mathrm{cm}^{3}\right)$

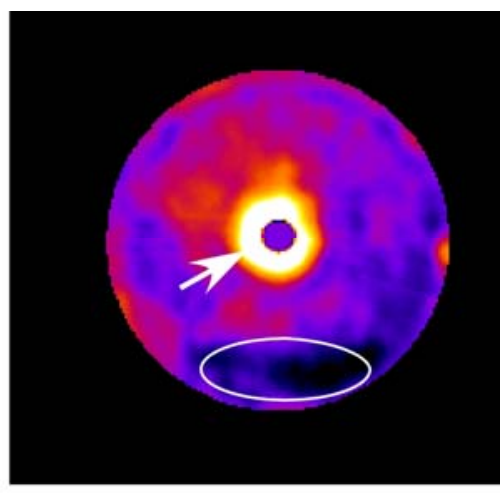

$\begin{array}{lllll}0.0 & .075 & .075 & .225 & .30\end{array}$

Density Change $\left(\mathrm{g} / \mathrm{cm}^{3}\right)$

Figure 8. One location in the excess water test. a. Density distribution of dry sample. Note low density (high porosity) region near the thermocouple (white dot) in the sample center (arrow). b. Density change upon adding water (Note large density change indicating higher water saturation in the oval, and low density change indicating remaining gas near the thermocouple), c. further density change upon hydrate formation (Note very little change in the initially well-saturated oval, and significant change from hydrate formation at the center.

\section{Conclusions}

Water imbibition into a dry sand does not distribute water and gas evenly. Resultant hydrate formation is dependent on the location of the trapped gas, thus the hydrate saturation is also heterogeneous. In our sample, it appears that both distributed and concentrated hydrate regions were formed.

\section{Unsaturated - Sand + Kaolinite}

\section{Description}

Hydrate was made using the excess gas method. The sample was composed of $99.5 \% \mathrm{~F}-$ 110 sand and $0.5 \%$ kaolinite. In this test, hydrate was formed with a mixed gas (66\% CO2, 34\% methane).

\section{Hypothesis}

Edges on kaolinite particles will provide nucleation sites for hydrate formation. If these are well distributed in the sample, hydrate will nucleate everywhere at once.

\section{Results}

Figure 9 shows the change in density upon hydrate formation, with red being increased density from hydrate formation and blue being decreased density (from water migration towards forming hydrate). 


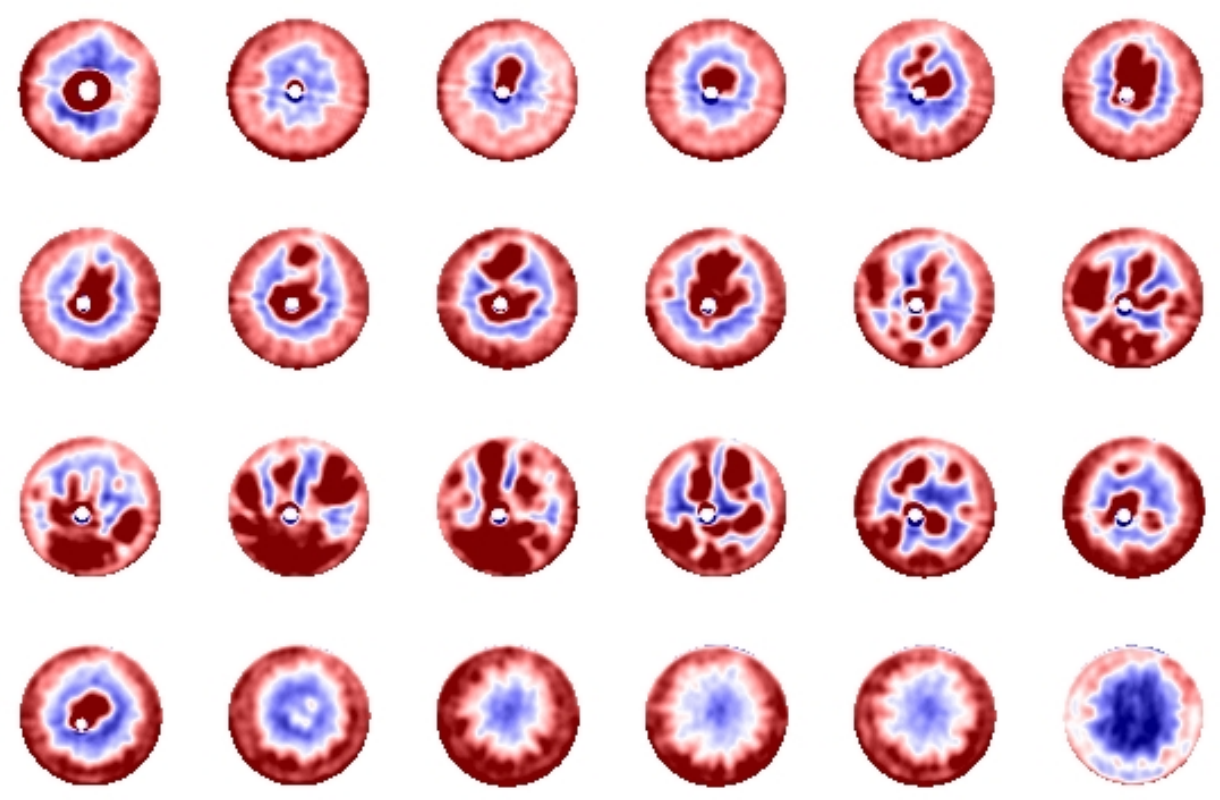

Figure 9. Changes in sample density upon hydrate formation. Increases in density from hydrate formation are shown in red, decreases in blue. The maximum density increase is about $0.2 \mathrm{~g} / \mathrm{cm}^{3}$, and the maximum density decrease is about $0.13 \mathrm{~g} / \mathrm{cm}^{3}$.

\section{Conclusions}

The addition of kaolinite to the sand did not aid in forming homogeneous hydrate. Because this test was performed with a mixed gas, it is not known whether the behavior of the system with a pure gas (methane) would be different.

\section{Use of a Nucleation Enhancing Agent}

\section{Description}

The excess gas method was used to create hydrate in a moist sand sample in which the water phase contains a nucleation enhancer (SnoMax). SnoMax was selected because it is often used as a nucleation enhancer for man-made snow at ski parks.

\section{Hypothesis}

The ubiquitous presence of a nucleation enhancer will cause hydrate to begin forming everywhere throughout the sample causing the sample hydrate saturation to be uniform.

\section{Results}

Several tests were performed using SnoMax as a nucleation enhancer. In the first test, the sample was poorly packed resulting in an initially heterogeneous packing density. In spite of this, the resulting density change indicated that hydrate was somewhat evenly distributed (Figure 10 - top); however some regions were depleted of water (blue). The test was repeated with a more homogeneously packed sample. In this test, hydrate preferentially formed along the center axis of the sample. The test was repeated with an even more homogeneously packed sample without the thermocouple, and again, hydrate was preferentially formed along the center axis. 

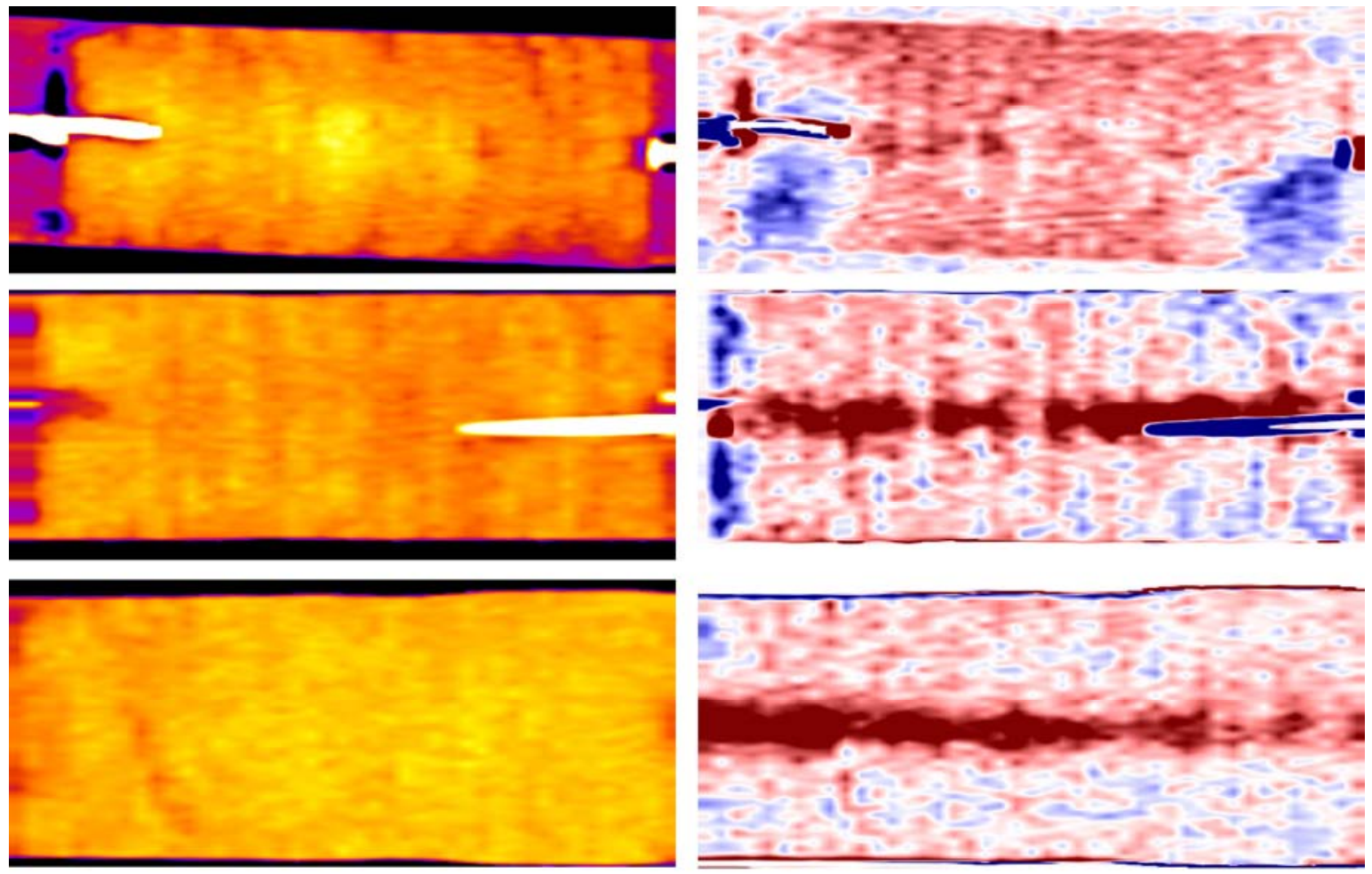

1.6517 .81 .92 .032 .15

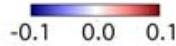

Density Change $\left(\mathrm{g} / \mathrm{cm}^{3}\right)$

Density $\left(\mathrm{g} / \mathrm{cm}^{3}\right)$

Figure 10. Axial CT cross sections. Left - initial sample density. Right - change in sample density upon hydrate formation. Top - Test 1, Middle - Test 2, Bottom Test 3.

In the middle and bottom tests in Figure 10, hydrate was formed by first pressurizing the sample before dropping the temperature. It was hypothesized after the third test (Figure 10-bottom) that the thermal gradient across the core was causing hydrate to form preferentially in the center. A final test was conducted where the hydrate was formed by pressurizing a chilled sample. This sample did not have hydrate preferentially along the center axis, however it was preferentially located towards one end of the sample, and water depleted from the other end.

\section{Conclusions}

The use of SnoMax to enhance nucleation had a varied effect on hydrate formation in F110 sand. When formed from pressurization it showed a tendency to form evenly across the X-Y axis of the core, but would leave patches of the sample devoid of hydrate formation. When formed by cooling, hydrate formed along the central axis of the sample. Overall. It appears that the addition of SnoMax to the pore water did not promote uniform hydrate formation in the samples.

\section{Use of Water Containing Salt}

\section{Description}


In this test, hydrate was formed using the excess gas method. Instead of pure water, a sodium chloride brine was used to moisten the sand.

\section{Hypothesis}

Salts are hydrate inhibitors and are excluded in hydrate formation. Thus hydrate forming in water containing salts will concentrate the salt making subsequent hydrate formation more difficult. The hypothesis behind this test was that if hydrate were to start forming a one location, the increase in salt concentration in this area would slow or stop the formation locally, allowing hydrate to form at other locations having more dilute salt concentrations resulting in an overall uniform hydrate distribution.

\section{Results}

A single test was performed using salty water $(10 \mathrm{~g} / \mathrm{L} \mathrm{NaCl}$ roughly a third of the concentration of sea water). The sand was packed relatively uniformly. The sample density changes following hydrate formation are shown in Figure 11. Hydrate formed preferentially along the sample axis as it did with the SnoMax. Unlike the SnoMax samples, hydrate formed predominantly in a certain region in the sample (See the red color towards the right in Figure 11) while depleting water from other regions (See the blue color towards the left of Figure 11.).

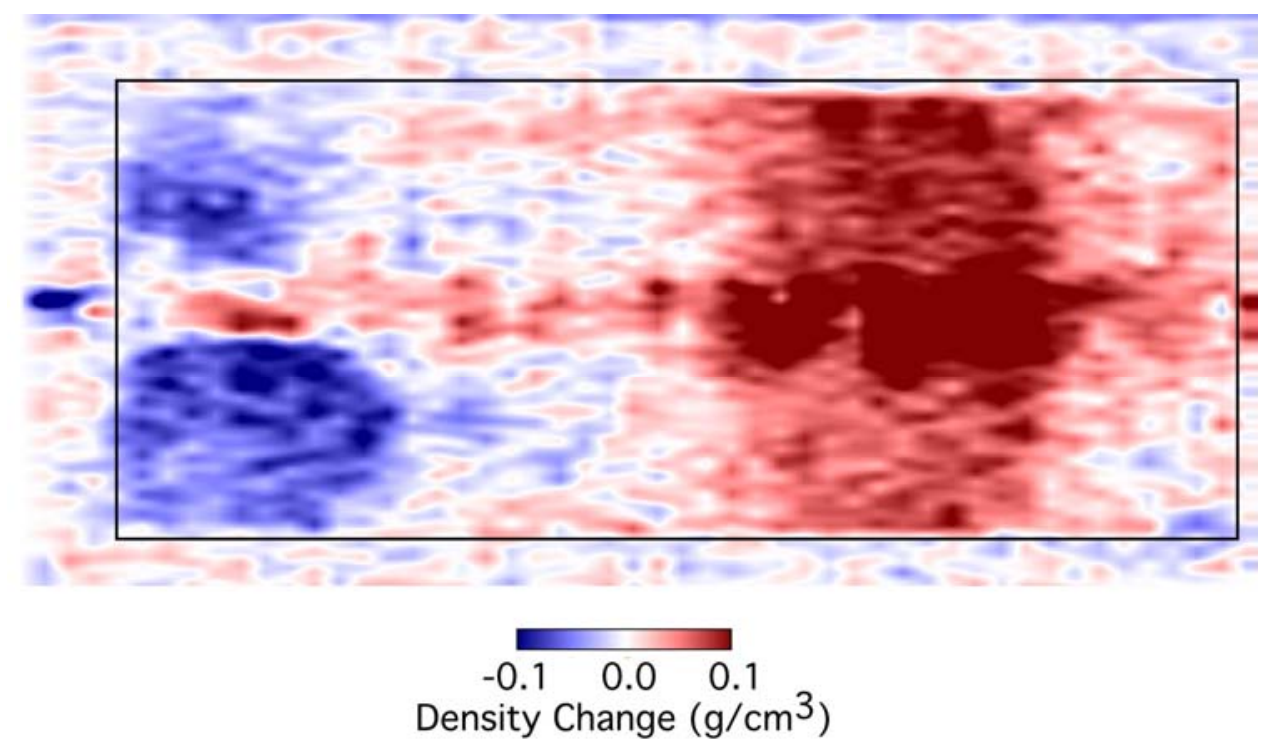

Figure 11. CT axial cross section of density changes in sample containing initially salty water. The black rectangle approximately outlines the sample. The red color indicates increases in density from hydrate formation, and blue indicates density decreases from water migration.

\section{Conclusions}

The hydrate-bearing sand sample produced in our laboratory test was not homogeneous and had one of the more heterogeneous hydrate distributions in our series of tests.

\section{Summary and Conclusions}

A large amount of data from fifty-five tests is presented here. In spite of the number of tests, only a limited number of hydrate formation influencing factors have been examined 
towards making representative hydrate-bearing samples containing a homogeneously distributed hydrate in sediment. Most of these tests were performed in the same pressure vessel and sleeve. This provides a standard by which the samples can be more fairly compared. This also applies a similar bias to many of the tests that may influence the results. For example, hydrate formation depends on heat transfer, and the heat transfer in many of these tests was governed by the system in which the hydrate was forming. In another system, behavior may be different.

The following methods were examined:

-Excess gas

-Freeze/thaw/form

-Freeze/pressurize/thaw

-Excess gas followed by water saturation

-Excess water

- Sand and kaolinite

-Use of a nucleation enhancer (SnoMax)

-Use of salt in the water

No method as of yet performs extremely well. The Freeze/Thaw/Form technique has so far tended to yield the most uniform samples, with one sand/water content routinely yielding heterogeneous samples (F-110, 35\% initial water saturation). We believe that the Freeze/Pressurize/Thaw method, if performed slowly, might yield even better results. It will still be influenced by saturation redistribution upon freezing, however more rapid freezing might reduce this effect.

The nucleation enhancers (SnoMax and kaolinite) we tried were not effective in producing homogeneous samples. Another enhancer that should be examined is bentonite clay, which behaves differently than the kaolinite [Uchida et al., 2004].

Water flooding a sample made using the excess gas method strongly affects the sample behavior, and made our cementing hydrate behave similarly to pore filling hydrate. Combining this method with another method that yields uniform hydrate may perhaps provide the uniform samples needed for investigation.

\section{References}

Gupta, A., et al. (2006), Composite thermal conductivity in a large heterogeneous porous methane hydrate sample, J Phys Chem B., 110(33), 16384-16392.

Handa, Y. P., and D. Y. Stupin (1992), Thermodynamic properties and dissociation characteristics of methane and propane hydrates in 70-.ANG.-radius silica gel pores, The Journal of Physical Chemistry, 96(21), 8599-8603.

Katsuki, D., et al. (2006), Formation, growth and aging of clathrate hydrate crystals in a porous medium, The Philosophical Magazine, 86(12), 1753-1761.

Katsuki, D., et al. (2007), Methane hydrate crystal growth in a porous medium filled with methane-saturated liquid water, The Philosophical Magazine, 87(7), 1057-1069. 
Kneafsey, T. J., et al. (2007), Methane Hydrate Formation and Dissociation in a CoreScale Partially Saturated Sand Sample, JPSE, 56, 108-126.

Minagawa, H., et al. (2005), Water permeability measurements of gas hydrate-bearing sediments, in Fifth International Conference on Gas Hydrates, edited, p. 1058, Trondheim, Norway.

Priest, J. A., et al. (2009), Influence of gas hydrate morphology on the seismic velocities of sands, J. Geophys. Res., 114.

Seol, Y., and T. J. Kneafsey (2009), X-ray computed-tomography observations of water flow through anisotropic methane hydrate-bearing sand, JPSE, 66(3-4), 121-132.

Spangenberg, E., and J. Kulenkampff (2005), Physical properties of gas hydrate-bearing sediments, in Fifth International Conference on Gas Hydrates, edited, p. 2028, Trondheim, Norway.

Stern, L. A., et al. (1996), Peculiarities of methane clathrate hydrate formation and solidstate deformation, including possible superheating of water ice, Science, 273(5283), 1843-1848.

Tohidi, B., et al. (2002), Application of high pressure glass micromodels to gas hydrates studies, in Fourth International Conference on Gas Hydrates, edited, pp. 761 - 765, Yokohama.

Uchida, T., et al. (2004), Decomposition of methane hydrates in sand, sandstone, clays, and glass beads, J. Geophys. Res., 109.

Waite, W. F., et al. (2004), Methane hydrate formation in partially water-saturated Ottawa sand, American Mineralogist, 89, 1202-1207.

Waite, W. F., et al. (2008), Physical property changes in hydrate-bearing sediment due to depressurization and subsequent repressurization, JGR, 113(B07102).

Yun, T. S., et al. (2007), Mechanical properties of sand, silt, and clay containing tetrahydrofuran hydrate, J. Geophys. Res., 112, B04106. 


\section{DISCLAIMER}

This document was prepared as an account of work sponsored by the United States Government. While this document is believed to contain correct information, neither the United States Government nor any agency thereof, nor The Regents of the University of California, nor any of their employees, makes any warranty, express or implied, or assumes any legal responsibility for the accuracy, completeness, or usefulness of any information, apparatus, product, or process disclosed, or represents that its use would not infringe privately owned rights. Reference herein to any specific commercial product, process, or service by its trade name, trademark, manufacturer, or otherwise, does not necessarily constitute or imply its endorsement, recommendation, or favoring by the United States Government or any agency thereof, or The Regents of the University of California. The views and opinions of authors expressed herein do not necessarily state or reflect those of the United States Government or any agency thereof or The Regents of the University of California.

Ernest Orlando Lawrence Berkeley National Laboratory is an equal opportunity employer. 